\title{
Interférences
}

Ars scribendi

$7 \mid 2014$

Le savoir sur la langue

\section{Noms et puissances assimilatrices des âmes}

Alain Lernould

\section{OpenEdition}

Journals

Édition électronique

URL : http://journals.openedition.org/interferences/4643

DOI : 10.4000/interferences.4643

ISSN : $1777-5485$

Éditeur

HiSoMA - Histoire et sources des Mondes antiques

Référence électronique

Alain Lernould, "Noms et puissances assimilatrices des âmes », Interférences [En ligne], 7| 2014, mis en ligne le 10 juillet 2014, consulté le 15 septembre 2020. URL : http://journals.openedition.org/ interferences/4643; DOI : https://doi.org/10.4000/interferences.4643

Ce document a été généré automatiquement le 15 septembre 2020.

Tous droits réservés 


\title{
Noms et puissances assimilatrices des âmes
}

\author{
Alain Lernould
}

Thémis ou Gaia, forme unique sous maints noms divers

(A., Pr., 209 sq.)

1 Le Cratyle de Platon traite de la question de savoir si les noms existent par nature ou par convention, et, pour dépasser cette opposition, Socrate a recours au couple conceptuel forme - matière. Le même Socrate défend aussi une thèse centrale dans le Cratyle : le nom est une imitation, par des lettres et des syllabes, de l'essence de l'objet (423e); le nom est une image (eikôn, 431c-433c). Tout cela est déjà comme annoncé dans ce passage qui se situe dans les premières pages du dialogue ( $\mathrm{Pl}$., Cra. 389d4-390a2) :

Eh bien, mon excellent ami, le nom qui est naturellement approprié à chaque objet, notre législateur ne doit-il pas savoir l'imposer aux sons et aux syllabes, et avoir les yeux fixés sur ce qu'est le nom en soi, pour créer et établir tous les noms, s'il veut faire autorité en cette matière? S'il est vrai que chaque législateur n'opère pas sur les mêmes syllabes, voici cependant ce qu'il ne faut pas oublier : tous les forgerons n'opèrent pas non plus sur le même fer en fabriquant pour le même but le même instrument; néanmoins, tant qu'ils lui donnent la même forme, même si ce n'est pas le même fer, l'instrument est bon, qu'on le fabrique chez nous ou chez les Barbares. N'est-ce pas? (trad. L. Méridier ; je souligne)

Le nom est une forme appliquée à une matière que constituent les sons et les syllabes. Le nom dans le langage des hommes est donc un composé, mais des deux composants, c'est la forme qui est déterminante, et ces formes multiples de noms, engagées dans cette matière acoustique elle-même variable et conventionnelle que sont les éléments du langage, procèdent d'un modèle unique, du nom en soi, ou encore du «nom naturel de chaque objet " $(390 \mathrm{e} 2 \mathrm{sq} \text {. })^{1}$, sur lequel se règle le nomothète dans sa production « linguistique » ou « verbale ». La justesse naturelle des noms usuels et comme produits par un art se fonde sur l'adéquation de ces noms à un modèle absolu, non particulier, non produit, toujours identique à lui-même et un, une forme en soi. Somme toute, un idéalisme platonicien, sans surprise. Notre texte d'ailleurs nous fait venir à l'esprit aux moins deux autres passages platoniciens fameux : un extrait du Timée (30c3 sq.), dans lequel Socrate dit que le Démiurge produit le monde sensible les yeux fixés sur un 
Modèle intelligible ; et un autre de la République (510d4-511a1), où Socrate explique que le géomètre certes fait ses démonstrations sur des triangles figurés et visibles mais a en fait en vue le triangle en soi qu'on ne peut voir qu'avec la pensée. Dans la République, Socrate nous invite à passer du multiple et du sensible à l'un et l'intelligible; dans le Cratyle, de la même manière, le multiple et le sonore sont reconduits à l'un et à la forme en soi. Et dans le Timée, l'assignation du monde sensible au rang de «copie » conduira Timée à déterminer réflexivement son traité de physique comme n'étant qu'un « mythe vraisemblable » (29d1) ou un « discours vraisemblable » (30b8).

Dans son commentaire sur le Cratyle ${ }^{2}$, Proclus reprend à son compte cette thèse platonicienne selon laquelle le nom est une «copie». Mais bien loin d'attacher à ce terme de " copie » une connotation péjorative, il lui attribue au contraire une très nette valeur positive. La production de copies que sont par exemple les noms est l'expression de la puissance de production et d'assimilation des âmes partielles que sont les âmes (raisonnables) humaines. Telle est la thèse affirmée d'emblée au tout début du commentaire, quand Proclus, conformément au schéma néoplatonicien canonique du commentaire exégétique, livre comme premier « point capital» dans son introduction le « but » (skopos) du Cratyle (Procl., in Cra., § 1, p. 1-9):

Le but du Cratyle est de montrer l'activité génératrice des âmes et leur puissance assimilatrice dans les êtres tout derniers; les âmes ont reçu en lot cette puissance de par leur essence et elles la montrent au moyen de la justesse des noms.

Mais puisque l'activité divisée des âmes passe souvent à côté du but approprié, ce que fait aussi la nature divisée, il peut tout naturellement y avoir aussi des noms qui soient indéterminés et qui circulent par hasard et spontanément, et il n'est pas vrai que tous les noms sont des rejetons d'une science intellective et qu'ils cherchent à atteindre une parenté avec les réalités intelligibles (je souligne).

Le premier paragraphe résume la première partie du Cratyle, c'est-à-dire l'entretien avec Hermogène qui défend la thèse selon laquelle les noms existent par nature. Et on reconnaît dans le second paragraphe la seconde partie du Cratyle où, contre Cratyle qui soutient que tous les noms qui sont vraiment des noms sont justes (428b-430a), Socrate fait valoir que les noms ne sont pas toujours justes, qu'ils peuvent être des imitations inexactes (430a-433c).

5 Proclus reprend plus loin de manière plus développée cette idée que la production des noms n'est qu'une des formes d'expression de cette puissance en l'âme de produire des copies $^{3}$. Et puisque la copie implique la ressemblance avec le modèle (ce qui n'est pas le cas du simulacre, phantasma $)^{4}$, cette puissance de produire des copies, cette eikastikê dunamis ${ }^{5}$, est aussi puissance "qui assimile les êtres inférieurs aux supérieurs et les formes emportées dans la composition aux formes plus simples ${ }^{6} »$. L'assimilation n'est d'ailleurs pas seulement celle des êtres inférieurs aux êtres supérieurs; elle est aussi celle de l'âme elle-même aux êtres supérieurs, et celle des êtres inférieurs à l'âme. La fabrication de statues de dieux et de démons relève de cette puissance d'assimilation, comme la production des noms. Mais cette dernière est production de copies en quelque sorte plus immatérielles que ne le sont des statues (Procl., in Cra. 19, 8-12) :

parce qu'elle veut faire venir à l'existence des copies qui ressemblent aux êtres, des copies qui soient d'une certaine manière immatérielles ${ }^{7}$ et qui soient les rejetons de sa seule essence rationnelle, elle produit au jour elle-même, en se servant de l' imagination linguistique (chrômenêi têi lektikêi phantasiai) comme d'une auxiliaire, les noms qui existent (je souligne).

6 L'idée que l'imagination linguistique (ou verbale) soit mobilisée comme auxiliaire dans la production des noms est tout à fait remarquable. Certes on peut ne voir ici qu'une 
simple forme d'expression de l'idée traditionnelle selon laquelle le discours "proféré " (logos prophorikos) reflète la pensée intérieure, le logos intérieur (logos endiathetos), comme un miroir. C'est ainsi que Plotin peut dire (Plot., Enn. IV, 3 [27], 30, 5-11) :

peut-être est-ce le langage (logos), accompagnement de la pensée, qui sera accueilli dans le champ de l'imagination. Car la pensée n'a pas de parties et, quand elle n'est pas encore, si l'on peut dire, sortie au dehors, elle est au dedans de nous à notre insu; c'est le langage qui, en la déployant et en la faisant passer du champ de la pensée à celui de l'imagination, montre la pensée comme dans un miroir, et lui vaut ainsi d'être perçue, de demeurer et de laisser un souvenir (trad. J. Pépin) 8 .

7 En même temps, on ne peut pas ne pas faire immédiatement le rapprochement avec ce que Proclus nous dit du rôle de l'imagination en géométrie. Le raison discursive (dianoia), peut-on lire dans son commentaire sur les Éléments d'Euclide, possède en ellemême les « raisons " mathématiques; ces " raisons " sont des raisons "essentielles", c'est-à-dire constitutives de l'essence de l'âme; en tant que "raison essentielle », le cercle en la dianoia est un, immatériel, sans parties, inétendu. Bref, il est une forme qui ne peut être l'objet que d'une saisie simple, non discursive, non figurée, quelque chose donc comme une intellection. Mais l'âme partielle humaine est trop faible pour voir sous un mode simple et unifié ces "raisons", aussi les déploie-t-elle dans la matière intelligible qu'est l'imagination. L'imagination joue ainsi le rôle de miroir sur lequel la dianoia « projette » ses raisons, leur donnant de ce fait étendue et visibilité ${ }^{9}$. D'un autre côté, en tant que matière intelligible, l'imagination ne retire rien à la précision des figures géométriques dont elle est le réceptacle, et donc n'empêche pas la géométrie d'être pleinement une science. Bien au contraire, elle est même la condition de possibilité de l'existence de la géométrie comme science, car la raison du cercle, en elle-même sans étendue, ne peut constituer un objet de science, de démonstration: comment par exemple bissecter un cercle qui n'est que raison pure, qui est immatériel, sans grandeur, sans étendue spatiale ${ }^{10}$ ? Ainsi, en même temps qu'elle pallie la faiblesse de la dianoia, l'imagination permet à celle-ci de déployer une connaissance discursive et authentiquement scientifique des objets géométriques (Procl., in Euc., p. 55, 3-6 Friedlein) :

En l'imagination, ou plus précisément avec la coopération de celle-ci, la dianoia déroule la connaissance des raisons mathématiques en se réjouissant de la séparation d'avec les sensibles et en trouvant que la matière imaginative convient bien pour être le réceptacle de ses propres formes.

8 Proclus ajoute que cette projection et extériorisation des raisons géométriques dans l'imagination, et d'une manière générale le déploiement d'une science géométrique, n'est pas une fin en soi. Fidèle à l'enseignement de Platon dans la République ${ }^{11}$, il insiste sur l'idée que le véritable objet du géomètre n'est pas le cercle figuré dans l'imagination mais la forme inétendue du cercle, le cercle un et sans partie qui est dans la dianoia en tant que " raison essentielle ». La projection des « raisons essentielles » est la condition de possibilité d'une remontée vers ces mêmes raisons, d'un dépassement de la discursivié pour atteindre, sous le mode psychique, une intellection de ces raisons ${ }^{12}$. En dernière analyse, l'introversion en vertu de laquelle l'âme revient à sa propre intériorité permet à cette âme de remonter jusqu'à la Forme intelligible, l'Universel dans l'Intellect du Démiurge ou Raison Démiurgique ${ }^{13}$.

9 Je ne vais pas ici, dans le cadre de cet article, aller plus avant dans une comparaison de l'imagination linguistique avec l'imagination en géométrie chez Proclus, et d'une manière plus large dans le néoplatonisme tardif. C'est là une étude qui constituerait en 
elle-même et à elle seule l'objet, pour le moins, d'un autre article. Je ne retiendrai que le point suivant. En géométrie, et dans la mesure où l'on ne prend en considération que la figure «projetée» depuis et par la dianoia, indépendamment du discours démonstratif (dit ou écrit) sur la figure, il n'y a pas place pour la convention. La figure du cercle projetée dans l'imagination par la pensée est une image de la forme du cercle comme "raison psychique» (" raison essentielle»), mais c'est une image qu'on peut bien dire être la réplique exacte de son modèle en ce sens que la participation à une matière intelligible ne brouille pas la forme du cercle en lui retirant toute sa pureté. En un mot, et si l'on s'en tient au cercle "projeté " par la dianoia (par opposition au cercle "abstrait» de réalités sensibles, qui lui retient en fait toute l'imperfection de son origine sensible), il ne peut y avoir en géométrie de figures imparfaites ${ }^{14}$. Il n'en va pas de même pour les noms, ce qui implique que l'imagination linguistique (ou verbale), quelle que soit sa fonction précise dans l'art qu'est la production de noms, ne peut être mise sur le même plan que l'imagination dans la science qu'est la géométrie; il faut, me semble-t-il, nécessairement situer l'imagination langagière à un rang inférieur de degré de connaissance par rapport à l'imagination en géométrie.

10 J'en reviens maintenant à la puissance assimilatrice des âmes. Cette puissance assimilatrice, qui est puissance parfaite, c'est-à-dire génératrice d'acte ${ }^{15}$, se déploie par la production de copies, comme par exemple la production de noms justes. Mais cette puissance assimilatrice que possèdent les âmes (raisonnables) individuelles humaines doit être rapportée à sa source, à savoir la propriété assimilatrice qui apparaît pleinement pour la première fois au degré des dieux hypercosmiques ${ }^{16}$. Certes cette puissance assimilatrice est présente à tous les degrés du divin. Elle est précontenue dans l'Intellect démiurgique et dans le Modèle Intelligible; bien plus, la similitude "se répand depuis le haut à partir des dieux eux-mêmes ${ }^{17}$ ", c'est-à-dire à partir des Hénades; et ce n'est pas "seulement dans les êtres immatériels, mais aussi dans les formes unies à la matière et qui sont emportées dans le changement, que la similitude des formes, par son immobilité, ramène dans le cercle de la génération la déviation irrégulière des êtres particuliers ${ }^{18} »$. Mais en tant que précontenue par exemple dans le Démiurge Intellectif, la similitude existe "sous le mode de la cause ${ }^{19}$ ", et, «dans les Hénades qui sont au-dessus du degré de l'Être ", elle a reçu (avec la dissimilitude) une " existence primordiale ${ }^{20}$. C'est pourquoi « on ne doit pas dire que le caractère assimilateur est à titre premier dans le Démiurge ${ }^{21} »$. La classe de dieux qui est définie par la propriété assimilatrice est, nous dit Proclus, celle des dieux hypercosmiques (Procl., Theol.Plat. VI, 3, p. 14, 17 sq.) :

C'est donc à partir de ce degré que viennent à l'existence à titre premier les différentes classes de copies. En effet, toute copie est produite selon la ressemblance à son modèle, et fait ressembler les inférieurs aux supérieurs et relie toutes choses par la similitude, cela convient surtout à ces dieux-là : car qui peut rendre semblable à leurs modèles le monde lui-même et tout ce qui est dans le monde, sinon cette classe hypercosmique des dieux ${ }^{22}$.

11 On reconnaît ici un geste proclien typique : la reconduction à sa source divine d'un attribut ou d'une propriété appartenant à une réalité située assez bas dans la hiérarchie des êtres. En l'occurrence, la puissance assimilatrice des âmes individuelles humaines est rapportée ici à son principe divin: les dieux hypercosmiques. Cette lecture théologique du Cratyle ne doit pas nous surprendre. Platon est, aux yeux de Proclus, une âme divine. La théologie qu'il délivre lui a été transmise par une autre âme divine, celle de Pythagore, qui l'a elle-même reçue d'Orphée ${ }^{23}$. Cette théologie platonicienne se 
trouve dans tous les dialogues de Platon, exposée sous des modes divers : symbolique, imagé, inspiré et dialectique (c'est-à-dire scientifique) ${ }^{24}$. Le Cratyle ne fait pas exception, bien au contraire. Proclus le considère comme étant un dialogue théologique majeur ${ }^{25}$.

Il n'est peut-être pas inutile, à ce propos, de rappeler ici que le Cratyle occupe un rang honorable, si l'on peut dire, dans l'ordre canonique néoplatonicien de lecture des douze dialogues retenus comme principaux dans l'œuvre de Platon. Cet ordre de lecture correspond à une hiérarchie des degrés de vertus. Après le Premier Alcibiade qui apprend à nous connaître nous-mêmes et constitue ainsi une introduction à la philosophie (la philosophie vraie qui est celle de Platon) viennent, selon un ordre ascendant : le Gorgias qui correspond aux vertus politiques (la raison en l'âme maîtrise le corps dont elle use comme d'un instrument); aux vertus cathartiques (l'âme se purifie des passions corporelles et se sépare du corps) correspond le Phédon; une fois purifiée l'âme peut se livrer pleinement à l'étude des êtres réellement êtres, séparés, immatériels : en un mot, les êtres divins ; elle exerce alors les vertus théorétiques et se saisit du divin d'abord dans les mots, avec le Cratyle, puis dans les concepts, avec le Théétète, puis dans les réalités elles-mêmes, en commençant par les réalités naturelles, en lisant le Sophiste et le Politique, pour en arriver aux réalités théologiques avec le Phèdre et le Banquet. Ce premier cycle d'études est couronné par la lecture du Philèbe, qui a pour objet le Bien universel et même, indirectement, le Bien absolu. Un second cycle parachève ce cursus, avec la lecture des deux dialogues "parfaits» que sont le Timée et le Parménide, le premier étant une synthèse de la physique de Platon, le second une synthèse de sa théologie ${ }^{26}$.

La conception hylémorphique du nom conduit Proclus à accomplir ce même geste de reconduction de choses relevant du monde de la génération (les mots produits par les âmes incorporées) à leur source transcendante, divine, éternelle.

Pour les néoplatoniciens tardifs, les mots procèdent des notions simples qui sont dans l'âme. Ces notions simples procèdent elles-mêmes des réalités. On a ainsi un système à trois termes : pragmata (réalités) - noêmata (notions ou formes en l'âme) - phônai ou lexeis (mots) ${ }^{27}$. Les mots (entendons, les mots signifiants) signifient les réalités par la médiation des notions car "dire une réalité suppose que l'on possède préalablement (dans l'âme) une notion de cette réalité ${ }^{28}$ ». Cette triade : réalités - notions - mots est explicitement thématisée dans les commentaires tardifs au traité aristotélicien des Catégories pour établir la juste définition du but (skopos) de ce traité et éclairer la fonction précise de ces mots au statut particulier que sont les catégories, ces mots simples à partir desquels sont construites les propositions qui entrent dans les syllogismes démonstratifs et constituent d'une manière générale le discours susceptible de vrai ou de faux ${ }^{29}$. Dans ce contexte, les « réalités » sont des réalités substantielles et le mot pragmata, comme c'est souvent le cas par ailleurs, désigne les réalités immatérielles, séparées ${ }^{30}$; en un mot, il s'agit de l'Intelligible, assimilé souvent aux Formes transcendantes qui sont dans l'Intellect divin ${ }^{31}$.

Quant aux concepts ou notions (noêmata), ils peuvent avoir deux origines. Ils peuvent être «abstraits » des réalités sensibles. Ils sont alors dits " postérieurs dans l'ordre de l'être » (husterogenê) en ce sens qu'ils n'ont même pas ce semblant d'être qu'ont les réalités particulières, contingentes, soumises au devenir ; ces universaux «abstraits " sont purement et simplement «sans substance » (anousioi). Ces universaux « logiques » sont ceux d'Aristote et aussi ceux des commentateurs alexandrins (Ammonius ou 
Olympiodore par exemple), qui avaient une préférence pour Aristote (la majorité des commentaires sur Aristote proviennent de l'École d'Alexandrie). Mais on a pu défendre aussi l'idée que ces notions viennent de la propre intériorité de l'âme, que l'âme les tire de son propre fonds. C'est ce que soutiennent, plus fidèles à Platon, les grands maîtres de l'École d'Athènes. C'est dans cet esprit que Proclus, on l'a vu rapidement, rejette l'abstractionnisme aristotélicien en géométrie. Pour lui, les objets du géomètre ne dérivent pas, par abstraction, des objets sensibles, mais sont des "rejetons de l'âme ${ }^{32}$ " en ce sens qu'ils sont le produit d'une projection dans l'imagination, par l'âme, de « raisons » innées. Ces formes mathématiques "psychiques» sont substantielles; ce sont des essences, immatérielles, sans parties, qui sont en elles-mêmes objets d'une saisie non pas discursive mais unitive. Trop faible pour voir « de manière repliée » ces « raisons » qu'elle possède, l'âme les projette dans l'imagination comme sur un miroir. Cette projection permet à l'âme de revenir alors d'abord à sa propre intériorité, aux logoi constitutifs de son essence, qui à leur tour servent de point de départ à une remontée vers leurs modèles que sont les Formes intelligibles, contenues sous un mode transcendant dans l'Intellect du Démiurge. Ce que Proclus dit du cercle ou du triangle géométriques vaut bien sûr aussi pour la forme du cheval par exemple, ou celle de l'homme, qui sont en l'âme sous un double mode, si l'on peut dire. Les notions en l'âme d'homme ou de cheval sont en effet des représentations qui procèdent des raisons (logoi), c'est-à-dire des formes psychiques « essentielles» du cheval ou de l'homme que l'âme, en tant qu'elle est "convertie vers l'intellect", possède en elle; mais « lorsqu'elle s'est détournée de l'intellect [...] l'âme a fait de ces raisons des images qui remplacent les modèles ". Ces images des raisons en l'âme sont les notions dont l'âme se contente quand elle s'est « écartée de la ressemblance avec l'intellect ${ }^{33}$ ". Et puisque les raisons psychiques essentielles sont elles-mêmes des images des réalités intelligibles que sont l'Homme-en-soi ou le Cheval-en-soi, on peut donc dire que les «notions » sont des images d'images.

Au niveau des « réalités » et des «concepts » ou «notions », il n'y a que des formes ${ }^{34}$. C'est seulement avec le mot en tant qu'il est proféré au moyen des organes physiques de la voix (comme la langue ou la trachée-artère) et qu'il articule une matière acoustique que la forme devient forme engagée dans une matière.

Mais il faut une cause efficiente, qui impose la forme à la matière. Cette cause est l'onomathète ou, plus exactement, la pensée de l'onomathète (Procl., in Cra., § 49, p. 16, $28-17,9)$ :

Aristote dit que le langage est signifiant, non pas comme instrument, mais par convention (car, dit-il ${ }^{35}$, il n'est en rien étonnant que, tandis que le son de la voix est par nature, comme l'est le mouvement du corps, les noms soient par convention, comme l'est la danse [...] Même ces choses (la langue, la trachée-artère, etc.) contribuent à la fabrication du nom en lui donnant sa matière, mais c'est surtout la pensée de l'onomathète qui parachève cette production; c'est elle qui met la matière en harmonie avec la forme c'est-à-dire le modèle de la manière qui convient (je souligne) ${ }^{36}$.

18 On a là une application au cas de la production des noms du schème cosmologique platonicien bien connu qu'on trouve dans le Timée, où Platon expose la genèse du monde sensible en termes de coopération de la Raison et de la Nécessité ${ }^{37}$. Maintenant, il est clair pour Proclus (et pour nous aussi) que le Démiurge (la Raison divine) règle sa 'création' du Monde sensible sur des Formes intelligibles qui sont des principes créateurs, certainement pas des concepts « logiques ». Mais qu'en est-il du nomothète, 
de la pensée du nomothète? "Pensée ", dans notre citation ci-dessus, traduit le mot dianoia. Sur quoi se règle cette pensée pour produire les noms? Sur une notion «abstraite » ou sur une notion qui procède d'une forme psychique essentielle du nom et qui garde par là une forme de substantialité ? Pour Proclus, diadoque à l'École d'Athènes, c'est bien sûr à partir des raisons constitutives de son essence que l'âme produit les notions qui serviront de modèles pour la production des noms. Les noms sont « les rejetons de sa seule essence rationnelle ${ }^{38}$ ». Les mots (signifiants et justes) sont les « rejetons d'une science intellective ${ }^{39}$ ». Par "science intellective» il ne faut pas entendre seulement une science (démonstrative) des réalités métaphysiques ${ }^{40}$; « intellective » ne détermine pas seulement l'objet de connaissance, mais aussi le mode de connaissance. La création de noms justes a son principe dans une intellection dans et par l'âme individuelle humaine de l'essence des choses, dans une saisie unitive (non discursive) des réalités. C'est pourquoi les noms peuvent atteindre une "parenté avec les réalités ${ }^{41}$ ». En dernière analyse, ce sont les Formes transcendantes contenues dans l'Intellect du Démiurge qui sont la source ultime d'où procèdent les noms. Aussi le nomothète peut-il être appelé, par analogie, «démiurge » (Procl., in Cra., §51, p. 20, 1-6) :

Le Démiurge Universel est, selon Platon, le tout premier forgeur de mots [...] Si donc le nomothète est analogue au Démiurge, n'est-il pas nécessaire qu'il doive avoir aussi l'autorité d'imposer les noms. C'est pour cette raison que Platon appelle (Cra. 389a) le nomothète 'démiurge' et le plus rare des démiurges.

Forger des noms (justes) revient ainsi à s'assimiler à Dieu (Procl., in Cra., § 51, p. 19, 4 sq.) :

C'est en vertu de cette puissance (sc. de produire des copies, comme par exemple les noms) que l'âme peut se rendre semblable aux êtres qui lui sont supérieurs: les dieux, les démons, les anges.

Cette fonction salvatrice du langage, qui a été créée pour permettre aux âmes déchues de revenir d'abord à leur propre intériorité, et par là aux réalités contenues dans l'Intellect divin transcendant, sera à nouveau fortement affirmée dans ce passage tiré du commentaire de Simplicius aux Catégories (Simp., in Cat. 13, 4-9) :

Le langage est la limite de l'activité de l'âme, et le propre des limites est de convertir vers les principes: c'est pourquoi le langage rassemble en une communauté de pensée les âmes qui se sont écartées de l'intellect et des êtres, et qui se sont distinguées les unes des autres, il les fait s'ajuster aux réalités, il les fait remonter vers l'intellect et les prépare non seulement à vouloir être sans langage, mais même à posséder des notions qui ne soient plus différentes des réalités (trad. Ph. Hoffmann) ${ }^{42}$.

21 J'ai déjà fait remarquer plus haut que Proclus attribue à l'étude des noms et à la géométrie une même fonction salvatrice ${ }^{43}$. Un très beau texte, tiré du commentaire sur Euclide, sera plus éloquent que toute paraphrase. Il s'agit de la toute fin du premier prologue à ce commentaire, où Proclus fait un éloge vibrant de la mathématique en expliquant l'origine du mot «mathématique » (Procl., in Euc. 46, 15 - 47, 8) :

Voilà ce qu'est « l'acquisition du savoir (mathêsis) », c'est «la réminiscence » (Phédon $73 \mathrm{~b} 5 \mathrm{sq}$.) des raisons éternelles qui sont dans l'âme; c'est pour cette raison qu'est appelée «mathématique» (mathêmatikê) la connaissance qui contribue tout particulièrement à nous faire nous ressouvenir de ces raisons. C'est donc ainsi qu'est montrée à partir de son nom la fonction de cette science < mathématique > : elle impulse notre connaissance innée, actualise notre pensée intuitive, purifie notre pensée discursive et fait apparaître les formes qui par essence sont en nous; elle nous débarrasse de l'oubli et de l'ignorance qui nous viennent de la génération et elle 
nous délivre des liens de l'irrationalité, grâce au Dieu ${ }^{44}$ qui véritablement préside à cette science, lui qui produit au jour ses dons intellectifs, emplit toutes choses de raisons divines, fait remonter nos âmes vers l'intellect et les tire comme d'un engourdissement profond ; par la recherche il les fait se convertir vers elles-mêmes, en les faisant accoucher il les perfectionne, et par la découverte du pur intellect il les conduit à la vie bienheureuse. En consacrant ainsi à ce Dieu cet écrit nous mettrons un terme à notre étude de la science mathématique en général (je souligne).

On peut dire, à la lumière de ce texte, que l'objet du géomètre n'est pas finalement le cercle, ou le triangle, mais l'âme. De la même manière, le Cratyle n'a finalement pas pour objet le langage, mais l'âme. Le but de la géométrie, comme le but du Cratyle, est en effet de nous faire revenir à notre propre intériorité, de créer les conditions d'une « introspection conceptuelle anagogique ${ }^{45}$ ».

\section{BIBLIOGRAPHIE}

\section{Textes anciens}

Procli Diadochi in Platonis Cratylum Commentaria, ed. G. Pasquali, Bibliotheca scriptorum Graecorum et Romanorum Teubneriana, Lipsiae, 1908.

Proclo, Lezioni sul Cratilo di Platone, introd., trad. e commento di Fr. Romano, Symbolon 7, Catania, 1989.

Proclus, On Plato Cratylus, transl. by Br. Duvick, Ancient Commentators on Aristotle, London, 2007.

Prolégomènes à la philosophie de Platon, texte établi par L. G. Westerink et trad. par J. Trouillard, avec la collab. de A. Ph. Segonds, CUF, Paris, 1990.

\section{Textes modernes}

ABBATE M. 2001, Dall'etimologia alla teologia. Proclo interprete del Cratilo, Casale Monferrato.

HADOT P. 1980, «Sur divers sens du mot pragma dans la tradition philosophique grecque ", in P. Aubenque (éd.), Concepts et catégories dans la pensée antique, Bibliothèque d'histoire de la philosophie 50, Paris, p. 309-319.

HIRSCHLE M. 1979, Sprachphilosophie und Namenmagie im Neuplatonismus. Mit einem Exkurs zu „Demokrit" B 142, Beiträge zur klassischen Philologie 96, Meisenheim am Glan, 1979.

HOFFMANN Ph. 1987, «Catégories et langage selon Simplicius. La question du skopos du traité aristotélicien des Catégories ", in I. Hadot (éd.), Simplicius, sa vie, son œuvre, sa survie. Actes du colloque international de Paris, 28 sept. - $1^{\text {er }}$ oct. 1985, Berlin - New York, p. 61-90. 
- 1999, « Les analyses de l'énoncé. Catégories et parties du discours selon les commentateurs néoplatoniciens ", in Ph. Büttgen, St. Diebler, M. Rashed (éds), Théories de la phrase et de la proposition de Platon à Averroès, Études de littérature ancienne 10, Paris, p. 209-248.

LERNOULD A. à paraître, « Imagination proclienne et schématisme kantien », Études platoniciennes.

O’MEARA D. J. 2000, « La science métaphysique (ou théologie) de Proclus comme exercice spirituel ", in A. Ph. Segonds, C. Steel (éds), Proclus et la théologie platonicienne. Actes du colloque international de Louvain (13-16 mai 1998) en l'honneur de H. D. Saffrey et de L. G. Westerink, Leuven Paris, p. 279-290.

PÉPIN J. 1982, « Linguistique et théologie dans la tradition platonicienne », Langages 16, 65, p. 91-116.

ROMANO F. 1987, « Proclo lettore e interprete del Cratilo », in J. PÉPIN, H.D. SAFFREY (éds), Proclus lecteur et interprète des Anciens. Actes du colloque international du CNRS, Paris (2-4 octobre 1985), Colloques internationaux du CNRS, Paris, p. 113-136.

SHEPPARD A. D. R. 1987, « Proclus' philosophical method of exegesis: the use of Aristotle and the Stoics in the Commentary on the Cratylus ", in J. PÉPIN, H. D. SAFFREY (éds), Proclus lecteur et interprète des Anciens. Actes du colloque international du CNRS, Paris (2-4 octobre 1985), Colloques internationaux du CNRS, Paris, p. 137-151.

STEEL C. 2007, «Proclus on Divine Figures. An Essay on Pythagorean-Platonic Theology », in M. Bonazzi, C. Lévy, C. Steel (éds), A Platonic Pythagoras. Platonism and Pythagoreanism in the Imperial Age, Diatribai 2, Turhout, p. 215-242.

TROUILLARD J. 1975, «L'activité onomastique selon Proclos », in De Jamblique à Proclus. Neuf exposés suivis de discussions, Vandouvres - Genève, 26-31 août 1974, Entretiens sur l'Antiquité classique 21, Genève, 1975, p. 239-255.

VAN DEN BERG R. M. 2008, Proclus' Commentary on the Cratylus in Context. Ancient Theories of Language and Naming, Philosophia antiqua 112, Leiden.

\section{ANNEXES}

\section{Annexe}

La hiérarchie du divin chez Proclus

L'UN

Les Hénades

LES DIEUX INTELLIGIBLES

$1^{\text {re }}$ triade : l'Un-étant = l'Être intelligible

$2^{\mathrm{e}}$ triade $:$ l'Éternité $=$ la Vie intelligible

$3^{\text {e }}$ triade $:$ le Vivant-en-Soi = Intellect intelligible = le Modèle

Les DIEUX INTELLIGIBLES-INTELLECTIFS

$1^{\text {re }}$ triade = le « Lieu supracéleste »

$2^{\mathrm{e}}$ triade $=$ le $«$ Ciel $»$

$3^{\mathrm{e}}$ triade $=$ la « Voûte subcéleste »

LES DIEUX INTELLECTIFS

$1^{\text {re }}$ triade $=$ les «Parents» (Kronos, Rhéa, Zeus le Démiurge) 
$2^{\mathrm{e}}$ triade = les « Dieux immaculés »

La septième divinité

LES DIEUX HYPERCOSMIQUES (= chefs et assimilateurs)

LES DIEUX HYPERCOSMIQUES-ENCOSMIQUES (les 12 Dieux du Phèdre)

LES DIEUX ENCOSMIQUES présidés par Dionysos (= célestes et sublunaires)

LES ÂMES DIVINES

\section{NOTES}

1. Voir aussi 390a3 sq. où il est question de « la forme requise de nom » pour tel ou tel objet.

2. Ce «commentaire » est une collection d'extraits rassemblés par un excerptor à partir de notes prises par un auditeur du cours de Proclus sur le Cratyle. L'édition de référence est celle de G. Pasquali, Procli Diadochi in Platonis Cratylum commentaria, Lipsiae, 1908. Nous disposons de deux traductions en langues modernes, l'une en italien de Fr. Romano, Proclo. Lezioni sul Cratilo di Platone, Symbolon 7, Catania, 1989, et l'autre en anglais de Br. Duvick, Proclus. On Plato Cratylus, Ancient Commentators on Aristotle, London, 2007. Pour ce qui est des études sur ce commentaire, je mentionnerai ici : Trouillard 1975, Hirschle 1979, Romano 1987, Sheppard 1987, Abatte 2001 et Van Den Berg 2008. Sur la philosophie du langage de l'Antiquité tardive, et plus particulièrement sur l'interprétation des Catégories d'Aristote, voir Hoffmann 1987 et 1999.

3. In Cra., § 51, p. 18, 29 - 19, 12.

4. Sur la distinction entre copie et simulacre, cf. Sophiste $235 \mathrm{~d}-236 \mathrm{~b}$.

5. In Cra., § 51, p. 18, 29

6. In Cra., § 51, p. 19, 2 sq.

7. Peut-être aussi que par «immatérielles» est donnée implicitement ici l'idée que le langage oral (sonore) est moins «matériel » que le langage écrit, qui laisse une trace matérielle, cf. par ex. D.H., Hiérarchie ecclésiastique I, 4, PG 3, 376 C : le langage « est certes corporel, mais pourtant plus immatériel du fait qu'il échappe à l'écriture». Sur la dévaluation du langage écrit par rapport au langage parlé, cf. bien sûr déjà Pl., Phdr., 274e-276a ; voir aussi Lettre VII, 344c-d.

8. Cf. Pépin 1982, p. 94 sq.

9. In Euc. 121, 5 sq.

10. In Euc. 49, 24 sq.

11. R. 510d4-511a1.

12. In Euc. 54, 27 - 56, 4.

13. In Euc. 46, 15 - 47, 8 (cité ci-dessous).

14. En termes kantiens, la figure géométrique «projetée » est presque un concept pur, cf. Kant, Critique de la raison pure, Introduction: "L'intuition en question (sc. en mathématique) peut ellemême être donnée a priori et se distingue donc à peine d'un simple concept pur » (E. Kant, Cuvres philosophiques, I, éd. publiée sous la dir. de F. Alquié, Bibliothèque de la Pléiade 286, Paris, 1980, p. 763 = E. Kant, Critique de la raison pure, trad., présentation et notes par A. Renaut, $3^{\mathrm{e} e ́ d}$. corrigée, G.F., Paris, 2006, p. 98). Sur le rapprochement entre l'imagination géométrique chez Proclus et le schématisme kantien, voir Lernould, à paraître.

15. Procl., Inst., prop. 78 Trouillard.

16. Cf. l'Annexe : « La hiérarchie proclienne du divin ».

17. Procl., Theol.Plat. VI, 3, p. 17, 7 sq. Saffrey-Westerink.

18. Procl., Theol.Plat. VI, 3, p. 17, 8-12.

19. Procl., Theol.Plat. VI, 3, p. 15, 14 sq.

20. Procl., Theol.Plat. VI, 3, p. 18, 9-11. 
21. Procl., Theol.Plat. VI, 3, p. 15, 24-26.

22. De la même manière, Proclus peut dire que la figure se déploie formellement pour la première fois au niveau de la troisième triade des dieux intelligibles-intellectifs. Dans l'Intellect Intelligible, troisième triade des dieux intelligibles, les figures sont contenues sous un mode unifié, et dans la première triade des dieux intelligibles-intellectifs, elles le sont sous le mode causal (sur ce point, voir Steel 2007, spécialement p. 224-233).

23. Cf. Procl., Theol.Plat. I, 5, p. 25, 26 - 26, 4.

24. Cf. Procl., Theol.Plat. I, 4.

25. Procl., Theol.Plat. I, 5, p. 24, 12-19 : «Et s'il faut parmi les nombreux dialogues choisir ceux qui nous manifestent le plus l'initiation aux mystères divins, je ne pourrais mieux faire que de mentionner le Phédon, le Phèdre, le Banquet et le Philèbe, et de leur adjoindre aussi le Sophiste, le Politique, le Cratyle et le Timée, car tous ces dialogues, dans leur totalité, si l'on peut dire, sont pleins de la science divinement inspirée de Platon ». Dans la section 166 de l'In Cratylum (p. 90, 24-27 Pasquali), Proclus limite la portée théologique du Cratyle : «Dans le Cratyle le grand Platon a pour but non pas de célébrer les ordres supérieurs, médians et inférieurs des dieux, mais seulement leurs propriétés telles qu'elles apparaissent dans leurs noms ». Il faut comprendre par là que le Cratyle, qui nous instruit sur le divin à partir des images que sont les noms des dieux, n'est pas le Parménide, le dialogue théologique par excellence, où Platon livre de manière scientifique sa théologie complète.

26. Voir Westerink, Trouillard, Segonds (éds) 1990, p. 39 sq.

27. Voir par ex. Simp., in Cat., p. 13-21 Kalbfleisch. Cette triade est déjà contenue in nuce, dans ce fameux passage au tout début du traité De l'interprétation, où Aristote distingue, en les hiérarchisant, quatre niveaux de réalités : «Les mots sonores sont les symboles des affections qui sont dans l'âme, et les mots écrits des symboles des mots sonores. Tout comme l'écriture n'est pas la même pour tous les hommes, les mots sonores ne sont pas non plus les mêmes; mais les affections de l'âme, dont les mots sonores sont directement des signes, sont les mêmes pour tous, et les choses (pragmata) dont les affections de l'âme sont des images, étaient déjà aussi identiques ». Les «affections de l'âme " peuvent bien sûr comprendre les concepts ou notions (noêmata). Le commentateur alexandrin Ammonius identifie explicitement les «affections de l'âme » avec les notions, cf. Ammon., in Int., p. 19, 16-18 Busse.

28. Ammon., in Cat. 9, 22 sq. Busse.

29. Voir Hoffmann 1987, p. $75 \mathrm{sq}$.

30. Voir Hadot 1980, p. 318-319.

31. La distinction à l'intérieur de l'Intelligible (de l'Être) entre trois niveaux - intelligible, intelligible-intellectif, intellectif - n'est pas toujours reprise dans les textes néoplatoniciens tardifs, dans un souci pédagogique de clarté dans l'exposition.

32. In Euc. 13, 22.

33. Voir Simp., in Cat. 12, 16 sq. Simplicius ne parle pas de forme du cheval ou de forme de l'homme. Il parle seulement de "réalités" (pragmata) dans le cadre de la triade réalités notions - mots. C'est moi qui introduis ici ces exemples.

34. La matière intelligible qu'est l'imagination en géométrie n'est pas la matière sensible. De même, les notions impliquent certes une faculté de représentation, mais les notions sont bien en l'âme.

35. Arist., Int. 17a1 sq.: "Toute proposition a une signification, non pas toutefois comme un instrument naturel mais, ainsi que nous l'avons dit, par convention ».

36. On retrouve la même idée par ex. chez Ammonius, in Int., p. 63, 7-18: « c'est pourquoi il n'est pas nécessaire que le langage soit par nature ; car rien n'empêche que les produits des facultés naturelles soient par convention, comme c'est le cas pour la danse; en effet, tandis que le mouvement local est une faculté naturelle en nous, la danse, bien qu'elle soit le produit de cette faculté, est par convention; car il appartient au danseur, en bougeant les mains de telle ou telle 
manière, de signifier, par exemple, Achille, en montrant et en faisant voir au moyen de figures tantôt un épisode, tantôt un autre, de sa vie. De la même manière donc que se mouvoir dans l'espace se fait par nature tandis que danser se fait par convention et selon une règle, et que le bois est par nature tandis que la porte existe par convention, eh bien c'est de la même manière qu'émettre un son se fait par nature tandis que signifier quelque chose - au moyen de noms, ou de verbes, ou de phrases composées à partir de ceux-là, lesquels, s'ils existent à partir du son vocal brut qui est comme leur matière, se voient donner une forme par notre esprit - se fait selon une règle, et non par nature " (je souligne).

37. Voir Pl., Ti.47e-48a : «La naissance du monde a eu lieu par un mélange qui réunissait la Nécessité et l'Intellect. Mais comme l'Intellect dominait la Nécessité, en lui persuadant de réaliser dans les meilleures conditions possibles la plupart des choses qui sont soumises à la génération, c'est de cette manière et dans ces conditions que notre univers fut, dès le principe, constitué par le truchement d'une nécessité ainsi dominée par une sage persuasion ».

38. In Cra. 19, 9 (cité ci-dessus).

39. In Cra. 1,7 sq. (cité ci-dessus).

40. Voir Van den Berg 2008, p. 97.

41. In Cra. 1, 8 sq. (cité ci-dessus).

42. «Dans l'Intellect, qui est les réalités elles-mêmes et où il n'est pas besoin de langage, les êtres et les notions des êtres sont une seule et même réalité » (In Cat. 12, 16-19).

43. Voir aussi Van den Berg 2008, p. 195.

44. Il s'agit d'Hermès.

45. J'emprunte cette expression à O'Meara 2000, p. 290.

\section{RÉSUMÉS}

Pour le Néoplatoniciens l'acte de nommer, et le langage en général, sont propres aux âmes humaines rationnelles tombées dans le devenir. L'activité onomastique procède de la divinité et en même temps nous reconduit à l'unité de l'Intellect transcendant. Proclus, dans son commentaire sur le Cratyle de Platon, et Simplicius, dans son commentaire sur les Catégories d'Aristote, soulignent cette puissance assimilatrice de l'activité onomastique, où l'imagination « linguistique » joue un rôle d'« auxiliaire », analogue à celui que joue l'imagination en géométrie.

For the Neoplatonists naming and language are activities proper to human rational souls fallen into the world of generation. The onomastic activity proceeds from Deity and at the same time convert us to the unique transcendent Intellect. Proclus, in his commentary on Plato's Cratylus, and Simplicius, in his commentary on Aristotle's Categories, put extra emphasis on this assimilative power of the onomastic activity, in which the "linguistic" imagination plays the part of an "auxiliary" cause, analogous to the one played by imagination in geometry. 
INDEX

Keywords : hypercosmic gods, imagination, language, Neoplatonism, names, noemata, assimilative power, logoi

Mots-clés : dieux hypercosmiques, imagination, langage, néoplatoniciens, noms, notions (noêmata), puissance assimilatrice, raisons (logoi)

nomsmotscles Ammonius Alexandrinus Grammaticus, Aristote, Denys d'Halicarnasse, Euclide, Olympiodore, Platon, Plotin, Proclus, Pythagore, Simplicius

\section{AUTEURS}

ALAIN LERNOULD

UMR 8163 « Savoirs, Textes, Langage », Universités Lille 3 et Lille 1 